\title{
Toward Monitoring of Blazars at Abastumani: Progress Report.
}

\author{
Omar M. Kurtanidze ${ }^{1}$ \\ Astrophysikalisches Institute Potsdam, An der Sternwarte 16, D-14482, \\ Germany \\ Maria G. Nikolashvili \\ Abastumani Observatory, 383762 Abastumani, Republic of Georgia
}

\begin{abstract}
We present a brief summary of the ongoing Abastumani Blazar Monitoring Program started in the May of 1997. More than 50 000 frames have been collected during 498 observing nights for about 50 blazars.
\end{abstract}

\section{Introduction}

Variability time scales have been derived for many blazars from monitoring programs which attain a time resolution of days and to years (Wagner\&Witzel 1995, Smith 1996). The best example of the international cooperation is the multiwavelength study of a selected blazars in the frame of OJ-94 project carried out during the last decade (Takalo et. al. 1994). We started systematic multiband optical monitoring of blazars at Abastumani Observatory in the May of 1997. The aim of the programme is to study short-term and long-term variability of blazars and their correlations with that in radio, $\mathrm{x}$-ray and $\gamma$-ray bands.

\section{The Program, Observations and Data Reductions}

Abastumani Observatory is located in the South-Western part of Republic of Georgia at a latitude of $41^{\circ} .8051$ and a longitude of $42^{\circ} .8254$ on the top of the Mt Kanobili $1700 \mathrm{~m}$ above mean sea level. The weather and seeing conditions are very good in Abastumani (150 nights per year, $30 \% \leq 1$ arcsec). The mean values of the night sky brightness are $B=22.0, V=21.2, R=20.6$ and $I=19.8$.

The Blazar Monitoring Program at Abastumani Observatory was started in the May 1997 and is carried out with ST-6 CCD $(375 \times 242,23 \times 27 \mu \mathrm{m})$ Imaging Camera attached to the Newtonian focus of the $70-\mathrm{cm}$ meniscus telescope $(1 / 3$, $14.9 \times 10.7$ sq. arcmin). List of target objects was compiled using Catalogues published by Véron-Cetty\& Véron (1993), Padovani\&Giommi (1995) and from Perlman\&Stocke et al. (1996). In the period from May 1997 to Nov 2000 during 498 observing nights about 50000 frames were collected. All observations

\footnotetext{
${ }^{1}$ Visiting Astronomer, on leave from Abastumani Observatory
} 
are perfomed in the combined filters of glasses, which match B, V (Johnson) and $R_{C}, I_{C}$ (Cousins) bands well. Reference sequences in the blazar fields are calibrated using the Landolt's equatorial standard fields (Landolt, 1992). The primary data analysis software systems used are IRAF, MIDAS and STARLINK instaled on PC (Linux 6.2, 200Mhz MMX, 64Mb, 1.3 and 6.4 Gbts). Many light curves have been obtained during the great outburst and post-outburst era of BL Lacertae, 3C66, AO 0235+164, 1ES 0502+67.5, 0716+714, OI 090.4, PKS 0829+046, OJ287, 1ES 1028+511, Mrk 421, S4 0954+65, 1156+295, ON 231, 3C 279, OQ240 and others. Part of the results were published (Kurtanidze et al. 1998, 1999; Nikolashvili et al. 1999a,b). All objects under study show the variations over $0.5 \mathrm{mag}$. The largest one is observed for $\mathrm{AO} 0235+164$ and is equal to $4.0 \mathrm{mag}$ and IDV to $0.4 \mathrm{mag}$.

Acknowledgments. We thank the SOC for the kind invitation and financial support. O.M.K. gratefully acknowledges the invaluable financial support of the Astrophisikalisches Institut Potsdam without which this Programm will never be conducted.

\section{References}

Kurtanidze O.M., Nikolashvili M.G., Takalo L.O., 1998, in: Tosti G., Takalo L., (eds.) Proc. OJ-94 Annual Meeting 1997, Multifrequency Monitoring of Blazars. Publ. Osservatorio Astron. Università di Perugia, Perugia, vol. 3, p. 193

Kurtanidze O.M., Richter G.M., Nikolashvili M.G., 1999, in: Raiteri C.M., Villata M. \& Takalo L.O (eds.) Proc. OJ-94 Annual Meeting 1999, Blasar monitoring towards the third millennium. Publ. Osservatorio Astron. di Torino, p.29

Landolt A.U., 1992, AJ104, 340

Nikolashvili M.G., Kurtanidze O.M., Richter G.M., 1999a, in: Raiteri C.M., Villata M. \& Takalo L.O (eds.) Proc. OJ-94 Annual Meeting 1999, Blasar monitoring towards the third millennium. Publ. Osservatorio Astron. di Torino, p.36

Nikolashvili M.G., Kurtanidze O.M., Richter G.M., 1999b, in: Raiteri C.M., Villata M. \& Takalo L.O (eds.) Proc. OJ-94 Annual Meeting 1999, Blasar monitoring towards the third millennium. Publ. Osservatorio Astron. di Torino, p.33

Padovani P., Giommi P., 1996, MNRAS277, 1477

Perlman E., Stocke J.T., Schachter J.F., et al., 1996, ApJS104, 251

Smith A., 1996, in: Miller R., Webb J., Noble J., (eds.) Blazar Continuum Variability, 3

Takalo L.O., Silanpää A., Kidger M., 1994, in: Kidger M., Takalo L.O. (eds.), Workshop on Intensive Monitoring of OJ 287. Tuorla Observatory reports no.174, 3

Véron-Cetty M.-P., Véron P., 1993, A Catalogue of Quasars and Active Nuclei $\left(6^{\text {th }}\right.$ Edition). ESO Scientific Report 13

Wagner S.J., Witzel A., 1995, ARA\&A 35, 607 